\title{
Allergic Fungal Sinusitis
}

\author{
Daniel P. Correll $\cdot$ Scott A. Luzi $\cdot$ Brenda L. Nelson
}

Received: 24 November 2014/Accepted: 17 December 2014/Published online: 24 December 2014

(C) Springer Science+Business Media New York (outside the USA) 2014

\begin{abstract}
A 42 year old male presents with worsening pain and an increase in thick chronic drainage of the left sinus. Image studies show complete opacification of the left frontal sinus, left sphenoid sinus, and the left maxillary sinus. The patient was taken to the operating room and tissue for microscopic evaluation was obtained. The microscopic findings were classic for allergic fungal sinusitis: areas of alternating mucinous material and inflammatory cell debris and abundant Charcot-Leyden crystals. Cultures were performed and the patient began steroid therapy and desensitization therapy.
\end{abstract}

Keywords Tide lines · Congestion · Sinus drainage · Desensitization therapy

\section{History}

A 42 year old male is followed by Otorhinolaryngology clinic for chronic sinus congestion and interval worsening of left-sided pain and pressure over the past 2 years with chronic thick drainage on the left. Prior treatment with antibiotics has only provided slight relief of his complaints. The patient also complains of swelling of his left eye. He denies numbness, fevers, chills, or pain with chewing.

Disclaimer The opinions and assertions expressed herein are those of the author and are not to be construed as official or representing the views of the Department of the Navy or the Department of Defense.

D. P. Correll $(\square)$ · S. A. Luzi · B. L. Nelson

Department of Anatomic Pathology, Naval Medical

Center San Diego, 34800 Bob Wilson Drive,

San Diego, CA 92134-5000, USA

e-mail: daniel.correll@med.navy.mil

\section{Radiology}

A non-contrast Landmarx helical CT was performed, which revealed a heterogeneous mass-like lesion in the left nasal cavity involving the left ethmoidal air cell complex. Bony demineralization and bone deficiency was noted in the cribriform plate, planum sphenoidale, and medial left orbital wall with tissue extension causing displacement of left medial rectus muscle. There was complete opacification of the left frontal sinus, left sphenoid sinus, and the left maxillary sinus (Fig. 1a, b).

\section{Diagnosis}

Given the aggressive nature of this patients sinus disease, he was taken to the operating room for left image-guided endoscopic frontal sinusotomy, total ethmoidectomy, sphenoidotomy, maxillary sinusotomy, nasal septoplasty and inferior turbinate submucosal reduction. Intraoperative findings included severe polyposis and large amounts of gross eosinophilic-appearing mucin filling the sphenoid, frontal and ethmoid cavities on the left. There was also a large dehiscence of the lamina papyracea, with intact periorbital anatomy. Tissue specimens were obtained, and consisted of tan-gray left middle meatal tissue, which measured $1.8 \times 1.1 \times 0.2 \mathrm{~cm}$ in aggregate. On H\&E, copious free-floating mucin lakes were seen along with areas of alternating mucinous material and inflammatory cell debris, giving the appearance of "tide lines" (Fig. 2c). Nuclear cellular debris was visualized in aggregations, and Charcot-Leyden crystals were abundant (Fig. 2b). A polyp was identified with inflammatory features but no abscess formation. A GMS stain (with valid controls) revealed fungal organisms (Fig. 2a). The case was signed out as 


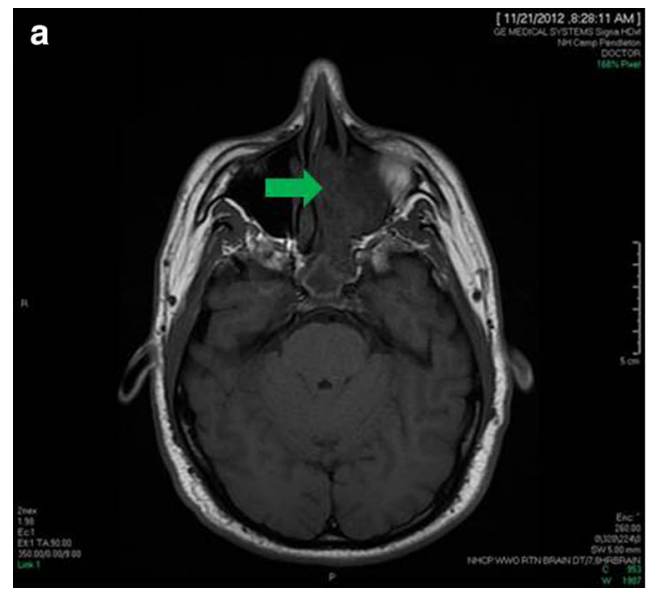

Fig. 1 a Magnetic resonance image (MRI) shows boney demineralization and an opacification of the left nasal cavity and sinuses. b Computed tomography (CT) also shows opacification of the sinuses,

inflamed sinonasal polyp and allergic fungal sinusitis (AFS). The bacterial cultures at the time were negative for an invasive bacterial component to this patient's condition. A fungal culture grew Penicillium spp. In addition, the patients serum IgE was markedly elevated at $515 \mathrm{kU} / \mathrm{L}$ (normal $<114 \mathrm{kU} / \mathrm{L}$ ), which is consistent with severe AFS. Postoperative CT scan revealed patent sinuses and open air cells, and the patient began steroid therapy and desensitization therapy at the allergy clinic.

\section{Discussion}

AFS, also known as Atopical Fungal Sinusitis or Eosinophilic Mucin Rhinosinusitis (EMRS), is an eosinophilpredominant IgE-mediated type I hypersensitivity reaction perpetuated by fungal allergens in the mucosa of the sinonasal tract. This results in tissue edema, and further proliferation of the fungal organisms increases antigen exposure and further inflammation resulting in chronic rhinosinusitis and sometimes polyposis [1, 3].

This condition occurs in immunocompetent people, with Aspergillus spp. being the most common culprit. Other fungal entities that have been associated are the brownpigmented dematiaceous fungi, Mucor, Penicillium, and many others [2]. AFS is a common entity, which may be seen in $10 \%$ of patients with polyposis or chronic rhinosinusitis. This entity is not seen in young children, and is most common in young adults (mean age of presentation is 21.9 years old) [4]. While gender distribution is equal, males typically have a higher incidence of bone erosion. The nasal cavity and all paranasal sinuses are subject to involvement, with the ethmoid and maxillary sinuses being the most frequent.

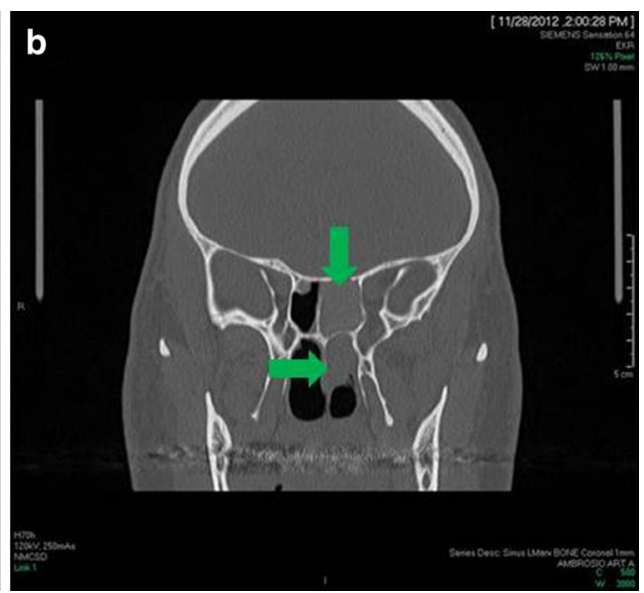

however, other entities were considered on the differential diagnosis to include sinonasal inflammatory polyps and a neoplasm

Clinical presentation of AFS may simply be chronic rhinosinusitis refractory to antibiotic therapy, or may be more dramatic, with some patients having a mass-like lesion and involvement of the orbit, causing proptosis or diplopia. Patients commonly describe thick rhinorrhea with a "greasy" or "muddy" consistency. Headache is also common. While peripheral eosinophilia may be noted on laboratory studies, along with IgE, these findings are not required for diagnosis and do not change management. Radiographic findings are commonly dramatic, with complete obliteration of involved sinuses, and may show bony erosion and a mass effect on computed tomography. [7]

Therapy for AFS is typically operative, with complete evacuation of inspissated mucus and debridement of involved tissue, as well as polypectomy if polyps are present. In addition to surgery, postoperative steroids are commonly used to reduce inflammation and allergy desensitization is recommended for long-term control. However, recurrences are common [3, 4].

Histologically, AFS has a characteristic appearance of "tide lines" or "tree rings" of mucin alternating with inflammatory debris, which appears as alternating pink and blue lines. The debris consists of aggregated eosinophils and neutrophils, and degenerated eosinophils forming Charcot-Leyden crystals. These crystals are refractile, and are long, bipyramidal, or needle-shaped. Mucinous material is free-floating. Any polyps that may be present are inflamed but do not have abscess formation. Fungal elements are difficult to identify on $\mathrm{H} \& \mathrm{E}$, and may be seen with GMS or PAS; if no fungal elements can be identified by staining or culture, then the entity may be diagnosed as Eosinophilic Mucin Rhinosinusitis, which confers the same prognosis and natural history and is considered to be synonymous with AFS $[1,5]$. 

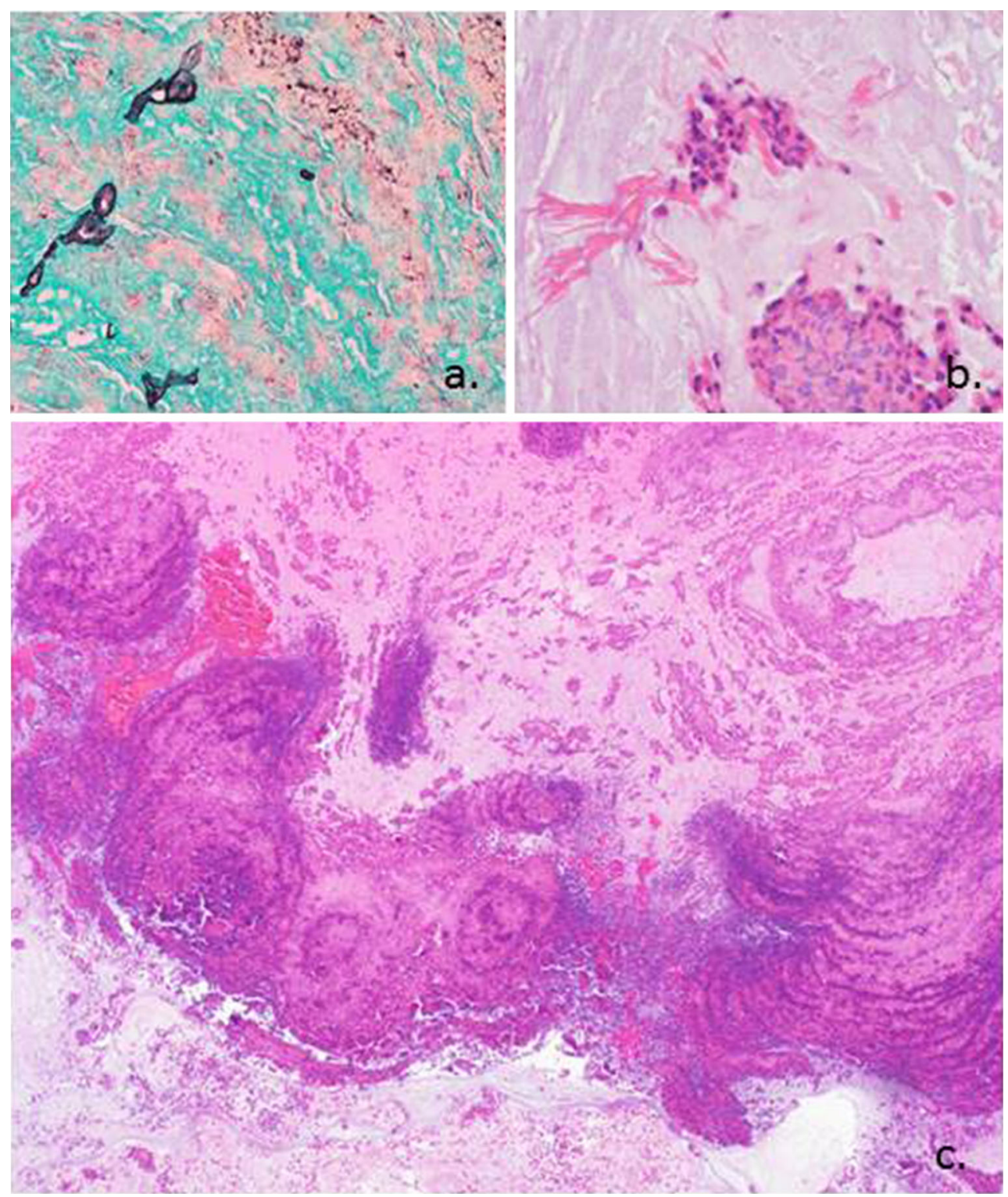

Fig. 2 a Grocott's methenamine silver (GMS) stain highlights the scant fungal elements seen in the specimen. $\mathbf{b}$ This high power image shows degenerated inflammatory cells and eosinophils with CharcotLeyden crystals. c Hematoxylin and eosin stain shows alternating

The differential diagnosis for this clinical presentation must be carefully considered, especially in situations involving periorbital involvement and bone erosion. Invasive Fungal Sinusitis (IFS) is treated much differently than AFS, and must be ruled out; the discriminating histopathologic feature of IFS is inflammatory cells within the tissue itself rather than free-floating in mucin; additionally, fungal elements are seen within the tissue and in vascular spaces. Another consideration is Sinonasal Polyposis, which is not associated with Charcot-Leyden crystals or the aggressive process seen with AFS. Finally, Mycetoma is a possibility as well; however, this would reveal an ripples of eosinophils and neutrophils creating a "tide line" or "tree ring" appearance. This low power picture is classic for allergic fungal sinusitis

aggregation of fungal elements with fruiting heads and minimal host response $[1,3,5,6]$.

\section{References}

1. Thompson, et al. Diagnostic pathology: head and neck. Manitoba: Amirsys Publishing Inc; 2011.

2. Arsenijevic, et al. Allergic fungal sinusitis-new aspects of clinical features, laboratory diagnosis and therapy. Srp Arh Celok Lek. 2013;141(9-10):698-704.

3. Huchton, et al. Allergic fungal sinusitis: an otorhinolaryngologic perspective. Allergy Asthma Proc. 2013;24(5):307-11. 
4. Marple MD. Allergic fungal rhinosinusitis: current theories and management strategies. Laryngoscope. 2001;111:1006.

5. Schubert: Allergic fungal sinusitis. Clin Rev Allergy Immunol. 1080-0549/06/205-216. 2006.

6. Driemal, et al. Allergic fungal sinusitis, fungus ball and invasive sinonasal mycosis - three fungal-related diseases. Mund Kiefer Gesichtschir. 2007;11(3):153-9.
7. Aribandi $\mathrm{M}$, et al. Imaging features of invasive and noninvasive fungal sinusitis: a review. Radiographics. 2007;27(5):128396. 